\title{
Dynamic Stochastic Optimization of Emergent Blood Collection and Distribution from Supply Chain Perspective
}

\author{
Xiangyu Jin $\mathbb{D}^{1,2}$ Huajun Tang $\mathbb{D}^{2}$ and Yuxin Huang ${ }^{2}$ \\ ${ }^{1}$ School of Logistics Management and Engineering, Zhuhai College of Science and Technology, Zhuhai, China \\ ${ }^{2}$ School of Business, Macau University of Science and Technology, Macau, China \\ Correspondence should be addressed to Huajun Tang; hjtang@must.edu.mo
}

Received 1 March 2021; Revised 5 April 2021; Accepted 12 April 2021; Published 24 April 2021

Academic Editor: Baogui Xin

Copyright (c) 2021 Xiangyu Jin et al. This is an open access article distributed under the Creative Commons Attribution License, which permits unrestricted use, distribution, and reproduction in any medium, provided the original work is properly cited.

In response to emergencies, it is critical to investigate how to deliver emergency supplies efficiently and securely to disasteraffected areas and people. There is no doubt that blood is deemed one of the vital relief supplies, and ensuring smooth blood delivery may substantially alleviate subsequent impacts caused by the disaster. Taking red blood cell products as the research object, this work proposes a four-echelon blood supply chain model. Specifically, it includes blood donors, blood donation houses, blood centres, and hospitals. Furthermore, numerical analysis is provided to test the feasibility of blood collection and distribution schemes and conduct sensitivity analysis to test the impacts of the relevant parameters (e.g., apheresis donation proportion of red blood cells (RBCs), distance between blood donors and blood facilities, and times of blood donation) on the scheme. This research provides some scientific and reasonable support for decision makers and managerial implications for emergency departments and contributes to the study of emergent blood supply chain.

\section{Introduction}

As multidimensional conflicts in politics, economy, and environment become increasingly evident, the world has witnessed an increase in the number of natural and manmade disasters. For instance, the terrorist attack in New York City on 11 September 2001 shocked the entire world and inspired other countries to pay more attention on emergency response. This was followed by a number of similar events, such as Wenchuan Earthquake in China in 2008, the earthquake and tsunami in Japan in 2011, the Nice terrorist attack in France in 2016, and mountain fire in Greece in 2018. These occurrences cause considerable human casualties and economic losses and impose dramatic negative impacts on people's psychological states and lives. In this context, the response to such complex emergent events requires a normative response-guaranteed system. As one of the critical materials for emergency rescue, the delayed supply of blood in an emergency poses threats to people's lives and health, whereas an excessive supply leads to waste and even subsequent "blood famine." This suggests the importance of incorporating a timely, adequate, and lowcost blood supply into emergency response and management decisions.

The study of emergent blood supply is one hot branch in the field of emergent management. There is an increasing number of literatures in emergent blood supply, most of which mainly focused on the downstream blood supply chain [1] and less of which investigated the blood collection and distribution from the perspective of the supply chain [2].

The aim of this study is to explore the optimal operations of a multinode and four-echelon blood supply chain in emergency, based on the status quo of emergency rescue in China. Specifically, given the low hazard level of emergencies, a four-echelon blood supply chain is established, including blood donors, blood donation houses, blood centres, and hospitals. Through numerical analysis, an optimal blood collection and distribution scheme is acquired for the blood supply chain. Sensitivity of the parameters (e.g., proportion of RBC apheresis donation, average volume of blood supply, number of blood donation, and distance 
between blood donors and blood facilities) is tested to explore the impacts on the optimal schemes.

The contribution of this work is to enrich the study of emergent blood collection and distribution in the field of emergent management and provide more scientific support for practical decision makers.

The remainder of this study is organized as follows. Section 2 summarizes relevant literatures on material supply and blood supply chain operations in response to emergencies. With respect to these literatures, it highlights that this work expands the previous research model of blood supply chain in several aspects, such as the selected targets, supply chain layers, dynamic stochastic demand, and blood collection and distribution models. In Section 3, a selfcollection model is established for the blood supply chain in response to emergencies. Section 4 conducts the numerical analysis so as to validate the feasibility of the proposed model. Finally, Section 5 concludes this study and provides some managerial implications.

\section{Literature Review}

Previous studies on emergency management can have two main categories with respect to the evacuation direction, including victim evacuation [3-5] and supply of emergency relief materials. Blood product is one type of emergency relief. In the following, it reviews the aspects of general material supply and blood product supply, respectively.

2.1. General Material Supply in Emergencies. Materials scheduling during emergencies covers emergency relief material distribution and transhipment routing. A multiperiod linear programming model was proposed for food aid layout optimization in Africa [6]. It aimed at minimising food transhipment and inventory costs. Goods delivery and victim evacuation were investigated under the circumstance that a limited number of transporting resources could be distributed to multiple destinations from diverse places [7]. This research involved three models with the goal of reducing the late deliveries. The earthquake in Turkey was taken for example to explore how the system cost could be minimised [8]. In the case where the supplied materials were limited and the demand for the material was known, they put forward an emergency supplies scheduling problem with a limited time window in emergency. Özdamar et al. [9] proposed a different model from traditional vehicle routing problems. Instead of returning vehicles that had completed their tasks to the place of departure and enabling them to wait there, the vehicles needed to wait for the next delivery command where they are. By combining network flow problems with vehicle routing issues, this research reduced the delay in scheduling emergency supplies to the greatest extent. Apart from that, a heuristic algorithm based on Lagrangian relaxation was adopted to solve the problem. To settle event response- and resource allocation-related issues in traffic accident management, a mathematical programming model with a probabilistic bound was developed. Yi and Özdamarb [10] was intended to investigate scheduling optimization problems in flood emergency rescue and elaborate and establish a flood emergency rescue programming framework, including demand, supply, inventory, and resource management. According to this framework, vehicles are considered as materials rather than variables. In subsequent studies, a heuristic algorithm of ant colony optimization was put forward to solve logistics problems in disaster-relief activities [11]. It decomposed the original emergency logistics into two stages. The first stage was to determine stochastic vehicle routing under the guidance of information elements, and in the second stage, the distribution of different vehicle types and various commodities were determined on the basis of network flow methods. Besides, a dynamic demand management model was proposed for emergency logistics operations under the circumstance that major natural disaster information conditions were incomplete [12]. Through fuzzy clustering, the disaster-affected area was divided into diverse groups. Then, the existing data were utilised to predict disaster-relief demands in different groups, and these demands were adopted as the basis of emergency supplies distribution. Yan et al. [13] determined that emergency supplies scheduling was correlated to disaster-affected road repair. For this reason, they selected seismic data from Taiwan in 1999 and took the randomness of vehicles' travel time into account. On this basis, a comprehensive optimization model was constructed for emergency supplies scheduling and disaster-affected road repair under uncertainty. It was expected that the cost incurred by accidental losses of after-calamity logistical support can be minimised.

Overall, emergency management is now proved challenging. Compared to conventional event management, emergency management needs to be studied further. The research on emergency management focuses on evacuation and supplies scheduling. In terms of emergency supplies scheduling, the existing literatures are primarily targeted at emergency scheduling of bulk supplies, but rarely related to perishable products, especially on blood products. Most of them did not take the characteristics of blood into account.

2.2. Blood Supply in Emergencies. Blood products fall into the category of important emergency relief supplies. This section presents a literature review of the studies associated with blood products. Blood supply chain includes blood collection, blood inspection and production, blood storage, and distributing the blood to those in need in a safe and timely manner. Previous studies focused on some of the following stages.

2.2.1. Collection. Blood collection, the first step in the whole blood supply chain operations, is one of the most fundamental tasks for mobile blood donation vehicles, blood donation houses, central blood stations, and blood centres. Red blood cells can be collected in two forms. One is whole blood collection and the other is apheresis collection, both of which are optional for donors.

Bosnes et al. [14] employed a logistic regression model to predict the arrival of blood donors in the blood bank. The 
multiobjective random integer linear programming model was used to determine an appropriate combined strategy of blood collection [15]. In order to minimise the total cost and the number of donors required, the model took factors such as the compatibility of blood types and the availability of blood types and donors into consideration. Concerning blood donation appointment scheduling, Seda et al. [16] utilised mixed-integer linear programming (MILP) to preassign blood collection periods for different blood types with the expectation of striking a balance between collection and production of different blood types on various dates. On this basis, they provided a steady blood supply system. Williams and Masser [17] evaluated the blood donation motivation of 458 qualified donors and concluded that motivation negatively impacts willingness to donate blood. Goette and Stutzer [18] carried out a large-scale field test for 3 months and designed a follow-up period of 15 months. In this way, they conducted preliminary experimental research on the influence of material incentives on blood donation. It was found that distributing rewards (e.g., holding lotteries) play a positive role in elevating the blood donation rate. Such an effect was driven by less enthusiastic donors.

According to the literatures above, research studies associated with blood collection mainly focused on the behaviour and motivations of donors, the estimation of donors' arrival, and donation appointments. In this work, blood collection and operation schemes are taken into consideration although they are seldom investigated in the existing studies.

2.2.2. Inventory Management. Most of the existing literature on blood inventory management focused on macro qualitative issues, whereas this research offers a summary and analysis of previous papers combining both qualitative and quantitative approaches.

By selecting RBC product inventory/disposal databases from provincial hospitals as the research object, Heddle et al. [19] made a logistic regression analysis of RBC inventorydisposal data over 21 months in 156 hospitals. This aimed at clarifying the factors that affected $\mathrm{RBC}$ inventory updates and systematically confirming an optimal target level of RBC inventory updates. Dillon et al. [1] suggested a two-stage stochastic programming model that minimised cost and expiration quantity and considered vulnerability and demand uncertainty. Moreover, a blood supply chain was constructed based on a blood ordering and collection model according to two emergency replenishment strategies [20]. These strategies considered heterogeneous requirements of blood and analysed the impacts of different emergency replenishment strategies on results.

2.2.3. Blood Distribution and Scheduling. This stage includes the distribution, transportation, and transhipment of blood products. Perishable product (i.e., blood) distribution strategies were analysed for different places, such as a regional centre, proposing two general policies based on optimal approximation [21]. To be specific, the above two policies were comprised of a rotation strategy and a retention strategy. Based on the existing distribution system of the Red Crescent blood service centre in Turkey, Sahin et al. [22] considered the influence of decision making about position on the performance of blood centres, stations, and moving units and established a mathematical model to solve decision making about central-location-based allocation concerning issues of blood supply.

Speaking of transportation, apart from an allocationrouting programming model (IAR) built based on multiple vehicles, multiple supply sites, and multiple objectives, an analytic hierarchy process and MILP were selected to settle relevant problems [23]. In addition, an integer programming model was constructed to identify the number of vehicles needed to implement blood collection operations and to minimise vehicles' travel distance [24]. The aforementioned model also took the uncertainty of blood donation vehicle visits into consideration and determined an optimal route by virtue of the CPLEX solver and the branch-and-price algorithm. Since 2017, the number of articles about blood transportation route programming in emergency relief has been in decline. In general, these topics were investigated in combination with other stages, such as blood collection, distribution, and inventory.

As for transhipment, Wang and Ma [25] achieved an effective reduction in the blood system expiration rate by establishing a transport model based on inventory storage time. Dehghani and Abbasi [26] created a transport strategy based on the longest storage time for interhospital blood transportation to reduce inventory scrap cost.

\subsubsection{Blood Supply Chain. In the last decade, there is in-} creasing research on blood products, especially on blood product operations in the supply chain [2].

For instance, Sha and Huang [27], taking Beijing as the background, developed an emergency blood supplyscheduling model, covering "donors, temporary blood donation facilities, and blood centres." Fahimnia et al. [28] built a four-echelon (i.e., blood donors, mobile blood donation sites, blood centres, and demand sites) blood supply chain model to minimise cost and delivery time. Ramezanian and Behboodi [29] introduced a deterministic location model based on MILP. Involving blood donors, mobile blood donation facilities, and blood centres, the model considered parameters such as the distance between blood donors and blood facilities and the advertising budget of blood facilities, which were used to constitute the utility function to improve usage and motivate blood donors. To minimise the total cost of collection, inventory, and production, Özener et al. [30] constructed a blood supply chain model with donors and central blood stations as nodes. By considering three different blood products, the model made plans for donors to meet the demand for blood products within a given range. Hamdan and Diabat [31] proposed a blood supply chain model based on robust optimization and two-stage stochastic optimization to minimise the impact of disasters on the blood supply chain. A dual-objective framework of simultaneous minimisation system was adopted to reduce the cost and delivery time during possible 
interruptions of the supply chain. According to the MILP model used by Samani et al. [32], various blood collection and separation methods and disruption scenarios were taken into consideration to make inventory and distribution decisions about the blood supply chain.

Literatures on blood supply chain operations with at least three stages during the last decade are listed in Tables 1 to 3 .

As can be observed from Table 1, previous research on blood supply chain with at least three echelons is rather rare in the past 10 years. Therefore, this is still a new research area and direction in this aspect. Blood supply chain studies involving all nodes at four levels and above are even fewer [33-38]. This work targets at a blood supply chain including donors, the blood donation house, the blood centre, and the hospital. Clearly, it is concerned with the entire blood supply chain.

In terms of objective functions, all studies incorporate the cost, while some of the literatures consider objective functions of blood shortage penalty and transhipment time. Few literatures added weight coefficients in different objectives $[39,40]$. In this study, the objective functions include the composite cost and the shortage penalty cost, both of which should be minimised; additionally, weight coefficients indicate the importance of different objectives. In the context of emergency, life safety is considered as the primary task in this work. Therefore, the cost incurred by expired products is excluded from the objective functions. Although the time factor is not counted as one of the objective functions, a rigid constraint for transhipment time is adopted as a constraint condition to ensure its urgency.

The present research is aimed at a four-echelon blood supply chain. Research on the operation of four-level blood supply chain can be traced back to 2017. By comparing different factors listed in Table 3, it is clear that almost all the literature on the four-level blood supply chain leaves various blood collection methods out of consideration. The research only refers to a simple method of whole blood collection. In this study, blood apheresis collection was also considered to explore how their variations affect results achieved based on a specific scheme. Compared with previous studies where blood donation houses were treated as nonadjustable fixed facilities, they are taken as adjustable candidate collection sites in this study and can be open or closed according to the actual situation. In the context of emergencies, this study also investigates how outcomes are affected by proportion of blood apheresis collection, the distance between blood donors and blood facilities, the average of the blood supply, and variations in the number of times of donation. A small amount of the existing literature involves sensitivity analysis but still attaches importance to the trade-off between objectives, the capacity of transport vehicles, and the variation of weight coefficients. This signifies that sensitivity analysis of the actual situation is still incomprehensive.

Overall, previous studies mainly focused on a single stage and rarely adopted the holistic perspective of supply chain. In this study, a blood supply chain model under emergencies is constructed, and the corresponding innovation can be summarized as follows.
(1) Most emergency relief supplies are materials in a broad sense and perishable products. Blood products are seldom investigated. However, this work is aimed at blood products and focused on their RBC products.

(2) From the perspective of the supply chain, studies on blood products turn out to be a single node or a supply chain of no more than three levels in most cases. The proposed model in this study consists of four stages. This expands the coverage of blood supply chain research and describes the status quo of blood supply chain operations.

(3) The present study takes optimization objectives into consideration comprehensively. To be specific, both the shortage cost and other composite operating costs are incorporated into the proposed model. Additionally, weight coefficients are introduced as the objective function to clarify importance of diverse objectives.

(4) Sensitivity analysis is conducted through altering the proportion of apheresis donation, times of blood donation, distance between blood donors and blood facilities and the average of the blood supply, etc. In this way, how parameters affect outcomes generated by the corresponding scheme can be explored.

(5) Compared with previous studies where blood donation houses were treated as nonadjustable fixed facilities, they are taken as adjustable candidate collection sites which can be open or closed according to the actual situation in this study.

\section{Problem Description and Model Formulation}

3.1. Problem Statement. Blood supply chain can be diverse in different countries or regions. In the following, China is taken as an example. A system of unified blood collection and supply institution planning and construction is implemented. Major units of blood collection, storage and inspection, and primary blood-use organisations are presented here.

(1) Blood centres are set up in provinces and municipalities directly under the Central Government, and central blood stations are set up in prefecture-level cities. As the two share similar functions, they are not distinguished in this work and are referred to collectively as "blood centres."

(2) Blood donation houses in each city are used for decentralised collection and preliminary testing of blood.

(3) As blood-use institutions, hospitals receive blood daily from the blood centres for clinical transfusion. Including four stages of "blood donor-blood donation house-blood centre-hospital," a blood supply 
TABLE 1: Supply chain echelon in some key literature.

\begin{tabular}{|c|c|c|c|c|c|c|}
\hline \multirow{2}{*}{ Literature } & \multirow{2}{*}{$\begin{array}{l}\text { Number of } \\
\text { stages }\end{array}$} & \multicolumn{5}{|c|}{ Node } \\
\hline & & Donor groups & Blood donation houses & Blood donation vehicles & Blood centres & Demand points \\
\hline Sha and Huang [27] & & $\sqrt{ }$ & $\sqrt{ }$ & - & $\sqrt{ }$ & - \\
\hline Jabbarzadeh et al. [33] & & $\sqrt{ }$ & $\sqrt{ }$ & - & $\sqrt{ }$ & - \\
\hline Arvan et al. [34] & & $\sqrt{ }$ & - & - & $\sqrt{ }$ & $\sqrt{ }$ \\
\hline Ramezanian et al. [29] & & $\sqrt{ }$ & $\sqrt{ }$ & - & $\sqrt{ }$ & - \\
\hline Osorio et al. [15] & 3 & $\sqrt{ }$ & - & - & $\sqrt{ }$ & $\sqrt{ }$ \\
\hline Hamdan and Diabat [31] & & - & - & $\sqrt{ }$ & $\sqrt{ }$ & $\sqrt{ }$ \\
\hline Haeri et al. [35] & & - & $\sqrt{ }$ & - & $\sqrt{ }$ & $\sqrt{ }$ \\
\hline Liu et al. [36] & & - & $\sqrt{ }$ & - & $\sqrt{ }$ & $\sqrt{ }$ \\
\hline Wang and Chen [37] & & - & $\sqrt{ }$ & - & $\sqrt{ }$ & $\sqrt{ }$ \\
\hline Fahimnia et al. [28] & & $\sqrt{ }$ & - & $\sqrt{ }$ & $\sqrt{ }$ & $\sqrt{ }$ \\
\hline Zahiri and Pishvaee [38] & & $\sqrt{ }$ & $\sqrt{ }$ & - & $\sqrt{ }$ & $\sqrt{ }$ \\
\hline Attari and Jami [39] & 4 & $\sqrt{ }$ & - & $\sqrt{ }$ & $\sqrt{ }$ & $\sqrt{ }$ \\
\hline Samani et al. [40] & & $\sqrt{ }$ & $\sqrt{ }$ & $\sqrt{ }$ & $\sqrt{ }$ & $\sqrt{ }$ \\
\hline Samani et al. [32] & & $\sqrt{ }$ & $\sqrt{ }$ & - & $\sqrt{ }$ & $\sqrt{ }$ \\
\hline This work & 4 & $\sqrt{ }$ & $\sqrt{ }$ & - & $\sqrt{ }$ & $\sqrt{ }$ \\
\hline
\end{tabular}

Note. Both blood donation houses and vehicles are designed for blood collection and preliminary blood tests only. They are deemed to be at the same level in this study.

TABLe 2: Objective functions in some key literature.

\begin{tabular}{|c|c|c|c|c|c|c|}
\hline \multirow{2}{*}{ Literature } & \multirow{2}{*}{ Number of stages } & \multicolumn{5}{|c|}{ Objective functions } \\
\hline & & Cost & Shortage & Expiry & Time & Weighting \\
\hline Sha and Huang [27] & & $\sqrt{ }$ & $\sqrt{ }$ & - & - & - \\
\hline Jabbarzadeh et al. [33] & & $\sqrt{ }$ & - & - & - & - \\
\hline Arvan et al. [34] & & $\sqrt{ }$ & - & - & $\sqrt{ }$ & - \\
\hline Ramezanian et al. [29] & & $\sqrt{ }$ & $\sqrt{ }$ & - & - & - \\
\hline Osorio et al. [15] & 3 & $\sqrt{ }$ & - & - & - & - \\
\hline Hamdan and Diabat [31] & & $\sqrt{ }$ & - & $\sqrt{ }$ & $\sqrt{ }$ & - \\
\hline Haeri et al. [35] & & $\sqrt{ }$ & - & - & - & - \\
\hline Liu et al. [36] & & $\sqrt{ }$ & $\sqrt{ }$ & - & - & - \\
\hline Wang and Chen [37] & & $\sqrt{ }$ & $\sqrt{ }$ & - & - & - \\
\hline Fahimnia et al. [28] & & $\sqrt{ }$ & - & - & $\sqrt{ }$ & - \\
\hline Zahiri and Pishvaee [38] & & $\sqrt{ }$ & $\sqrt{ }$ & - & - & $\sqrt{ }$ \\
\hline Attari and Jami [39] & 4 & $\sqrt{ }$ & - & - & $\sqrt{ }$ & - \\
\hline Samani et al. [40] & & $\sqrt{ }$ & - & - & - & - \\
\hline Samani et al. [32] & & $\sqrt{ }$ & $\sqrt{ }$ & $\sqrt{ }$ & - & - \\
\hline This work & 4 & $\sqrt{ }$ & $\sqrt{ }$ & - & $\sqrt{ }$ & $\sqrt{ }$ \\
\hline
\end{tabular}

TABLE 3: Factors considered in some key literature.

\begin{tabular}{lcccc}
\hline Literature & $\begin{array}{c}\text { Sensitivity } \\
\text { analysis }\end{array}$ & $\begin{array}{c}\text { Different collection } \\
\text { methods }\end{array}$ & $\begin{array}{c}\text { Blood donation houses open or } \\
\text { closed }\end{array}$ & $\begin{array}{c}\text { Context of } \\
\text { emergencies }\end{array}$ \\
\hline Fahimnia et al. [28] & $\sqrt{ }$ & - & - & $\sqrt{ }$ \\
Zahiri and Pishvaee & $\sqrt{ }$ & - & - & - \\
[38] & - & - & - & - \\
Attari and Jami [39] & - & - & - & $\sqrt{ }$ \\
Samani et al. [40] & - & $\sqrt{ }$ & $\sqrt{ }$ & $\sqrt{ }$ \\
Samani et al. [32] & $\sqrt{ }$ & $\sqrt{ }$ & \\
This study & & & - & \\
\hline
\end{tabular}


chain model is established to minimise comprehensive costs such as shortage cost of blood centre and opening cost of blood donation houses and so on. The model is expected to make the following decisions including

(1) Location of blood donation.

(2) Quantity of blood donated by donors at each facility.

(3) When and which blood donation house should be open.

(4) The quantity of blood assigned from blood centres to hospitals in the affected area. In addition, it also tests the impacts of the proportion of apheresis donation, times of blood donation, distance between blood donors and blood facilities, and the average of the blood supply chain on the decisions and the objective, respectively.

\subsection{Model Assumptions}

(1) Hospitals are assumed to be incapable of storing blood, and all blood for clinical use in these hospitals needs to be deployed by a blood centre.

(2) The location of blood donation houses is fixed, and the blood donation houses are in charge of transporting blood to blood centres in phases.

(3) Averages of both blood supply and demand are known in the affected area, and supply and demand within the research period both conform to Poisson distribution [25].

(4) A person can only donate one unit of blood (i.e., $200 \mathrm{ml}$ of blood and referred to as $1 \mathrm{U}$ below) at a time.

(5) The blood inspection failure rate can be ignored.

(6) Automobiles within the affected area are assumed to be the vehicles for blood delivery.

3.3. Definition of Parameters and Variables. The sets, parameters, and variables involved in the model are defined as follows.

(1) Definition of sets

$I$ : the set of donors, $i \in I$

$J$ : the set of candidate sites for blood donation houses, $j \in J$

$K$ : the set of blood centres, $k \in K$

$H$ : the set of hospitals, $h \in H$

$T$ : the set of rescue periods (one day is a period in this study), $t \in T$

(2) Definition of parameters

q: number of blood donation houses

$U$ : penalty cost of shortage

OJ: unit collection and preliminary test cost of whole blood collection

$O J^{\prime}$ : unit collection and preliminary test cost of apheresis collection
$O L$ : unit in-depth test and production cost of whole blood collection

$O L^{\prime}$ : unit in-depth test and production cost of apheresis collection

$T J$ : unit transportation cost of vehicle

WH: unit blood inventory maintenance cost

$D_{h t}$ : hospital demand in hospital $h$ at time $t$

$n$ : limit of blood donation times

$b$ : maximum storage capacity of blood donation houses

$c b$ : maximum storage capacity of blood centres

$S_{i t}$ : the maximum number of donor group $i$ at time $t$

$\beta$ : proportion of apheresis blood donation to total blood donation

$A_{i j}$ : distance from donor group $i$ to blood donation house $j$

$A_{i k}$ : distance from donor group $i$ to blood centre $k$ $A_{j k}$ : distance from blood donation house $j$ to blood centre $k$

$A_{k h}$ : distance from blood centre $k$ to hospital $h$ $A C$ : maximum distance acceptable to the donor $L T_{1}$ : time requirement of blood delivery from blood centre to hospital

$V_{c}$ : vehicle transport speed

$N$ : large value

Rather than the individual behaviour of blood donation, this study focuses on the homogeneity of group blood donation after emergency, as such, the group is taken as the research object.

(3) Definition of variables

$P_{j t}: 0$-1 variable; it is 1 if the blood donation house $j$ is open, and 0 if not.

$Y_{i j t}: 0-1$ variable; it is 1 if the donor group $i$ goes to blood donation house $j$ at time $t$ for donation, and 0 if not.

$Y_{i k t}: 0-1$ variable; it is 1 if the donor group $i$ goes to blood centre $k$ at time $t$ for donation, and 0 if not. $Y_{i j k t}: 0-1$ variable; it is 1 if the donor group $i$ goes to blood donation house $j$ at time $t$ for donation and delivers blood to blood centre $k$, and 0 if not. $Y_{k h t} 0$-1 variable; it is 1 if blood centre $k$ delivers blood to hospital $h$ at time $t$, and 0 if not.

$Q_{i j k t}$ : the amount of blood delivered to blood centre $k$ at time $t$, donated by donor group $i$ in blood donation hospital $j$.

$Q_{i k t}$ : the amount of blood donated by donor group $i$ at time $t$ to blood centre $k$.

$Q_{k h t}$ : the amount of blood delivered from blood centre $k$ to hospital $h$ at time $t$.

$I B_{k t}$ : the beginning inventory quantity of blood centre $k$ at time $t$.

3.4. Model Formulation. The operation process of the blood supply chain model in this study is shown in Figure 1.

3.4.1. Objective Functions. In view of the role that blood products play in emergency management, the shortage of 


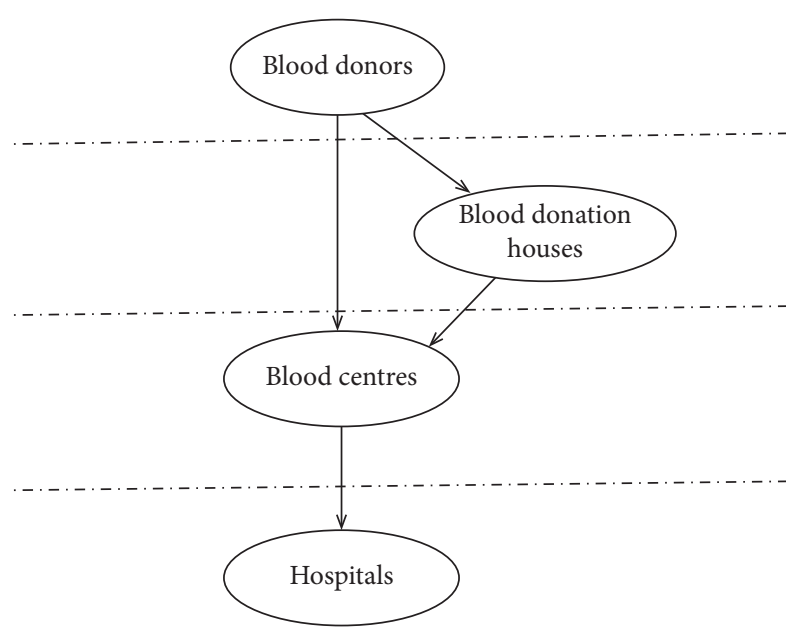

FIgURE 1: Operation chart of blood supply chain.

blood supply chain should be minimised. The shortage cost is shown in the following equation:

$$
Z_{1}=U * \sum_{h=1}^{H} \sum_{t=1}^{T}\left(D_{h t}-\sum_{k=1}^{K} Q_{k h t}\right),
$$

where $U$ is the penalty cost of shortage; $D_{h t}$ is the demand of hospital $h$ at time $t ; Q_{k h t}$ is the amount of blood delivered from blood centre $k$ to hospital $h$ at time; and $\sum_{h=1}^{H} \sum_{t=1}^{T}\left(D_{h t}-\sum_{k=1}^{K} Q_{k h t}\right)$ represents the amount of shortage of blood supply chain.

Moreover, blood supply chain operation should pay attention to cost control. The cost target is shown in the following equation:

$$
Z_{2}=\mathrm{PC}+\mathrm{OC}+\mathrm{TC}+\mathrm{IC},
$$

where PC is the opening cost of blood donation house; OC is the operating cost of blood collection and test; TC is the transportation cost; $X$ is the upward rounding of value $X$ in the symbol; and IC is the storage cost, and the formula is as follows.

$$
\begin{aligned}
\mathrm{PC}= & \mathrm{OP} * \sum_{j=1}^{J} \sum_{t=1}^{T} P_{j t}, \mathrm{OC}=\frac{2 \beta}{1+\beta}\left(O J^{\prime}+O L^{\prime}\right)\left(\sum_{i=1}^{I} \sum_{j=1}^{J} \sum_{k=1}^{K} \sum_{t=1}^{T} Q_{i j k t}+\sum_{i=1}^{I} \sum_{k=1}^{K} \sum_{t=1}^{T} Q_{i k t}\right) \\
& +\frac{1-\beta}{1+\beta}(\mathrm{OJ}+\mathrm{OL})\left(\sum_{i=1}^{I} \sum_{j=1}^{J} \sum_{k=1}^{K} \sum_{t=1}^{T} \mathrm{Q}_{i j k t}+\sum_{i=1}^{I} \sum_{k=1}^{K} \sum_{t=1}^{T} Q_{i k t}\right), \\
\mathrm{TC}= & 2 * T J\left[\sum_{k=1}^{K} \sum_{j=1}^{J} A_{j k}\left(\sum_{t=1}^{T} \frac{\sum_{i=1}^{I} Q_{i j k t}}{100}\right)+\sum_{k=1}^{K} \sum_{h=1}^{H} A_{k h}\left(\sum_{t=1}^{T} \frac{Q_{k h t}}{100}\right)\right], \\
\mathrm{IC}= & W H \sum_{k=1}^{K} \sum_{t=1}^{T}\left(I B_{k t}-\sum_{h=1}^{H} Q_{k h t}\right) .
\end{aligned}
$$

The overall objective function is shown in equation (4), where $w_{1}$ and $w_{2}$ are, respectively, the weight coefficients corresponding to the shortage cost target and other cost target, representing the weight emphasis of comprehensive measurement of each target.

$$
\min Z=w_{1} Z_{1}+w_{2} Z_{2} .
$$

$$
\begin{gathered}
\sum_{j=1}^{J} Y_{i j t}+\sum_{k=1}^{K} Y_{i k t} \leq 1, \quad \forall i \in I, \forall t \in T, \\
\sum_{t=1}^{T} \sum_{j=1}^{J} Y_{i j t}+\sum_{t=1}^{T} \sum_{k=1}^{K} Y_{i k t} \leq n, \quad \forall i \in I, \\
\sum_{j=1}^{J} P_{j t} \leq q, \quad \forall j \in J, \forall t \in T, \\
Y_{i j t} \leq P_{j t}, \quad \forall i \in I, \forall j \in J, \forall t \in T, \\
Y_{i j k t} \leq Q_{i j k t} \leq N * Y_{i j k t},
\end{gathered}
$$$$
\sum_{k=1}^{K}\left(\sum_{j=1}^{J} Q_{i j k t}+Q_{i k t}\right) \leq 1+\beta S_{i t}, \quad \forall i \in I \forall t \in T,
$$$$
\sum_{i=1}^{I} \sum_{k=1}^{K} Q_{i j k t} \leq b, \quad \forall j \in J, \forall t \in T,
$$

$\forall i \in I, \forall j \in J, \forall k \in K, \forall t \in T$, 


$$
\begin{aligned}
& Y_{i j k t} \leq Y_{i j t} \forall i \in I \forall j \in J \forall k \in K \forall t \in T \\
& Y_{i k t} \leq Q_{i k t} \leq N * Y_{i k t}, \quad \forall i \in I, \forall k \in K, \forall t \in T, \\
& Y_{k h t} \leq Q_{k h t} \leq N * Y_{k h t}, \quad \forall k \in K, \forall h \in H, \forall t \in T, \\
& A_{i j} * Y_{i j t} \leq A C, \quad \forall i \in I, \forall j \in J, \forall t \in T \\
& A_{i k} * Y_{i k t} \leq A C, \quad \forall i \in I, \forall j \in J, \forall t \in T, \\
& I B_{k t}-\sum_{h=1}^{H} Q_{k h t}+\sum_{i=1}^{I}\left(\sum_{j=1}^{J} Q_{i j k t}+Q_{i k t}\right)=I B_{k t+1} \text {, } \\
& \forall k \in K, \forall t \in T \text {, } \\
& I B_{k t} \leq c b, \quad \forall k \in K, \forall t \in T \\
& \frac{A_{k h} Y_{k h t}}{V_{c}} \leq L T_{1}, \quad \forall k \in K, \forall h \in H, \forall t \in T, \\
& D_{h t} \geq \sum_{k=1}^{K} Q_{k h t}, \quad \forall h \in H, \forall t \in T, \\
& I B_{k t} \geq \sum_{h=1}^{H} Q_{k h t}, \quad \forall k \in K, \forall t \in T .
\end{aligned}
$$

Equation (5) indicates that the quantity of blood collected at time $t$ should not exceed the maximum blood supply quantity limit at time $t$ in the area. Equation (6) expresses that the total amount of blood collected at time $t$ in the blood donation house will not exceed the volume of $j$. Equation (7) shows that group $i$ should only choose one of the locations $j$ or $k$ for donation at the same time. Equation (8) calculates the limit of donation times. Equation (9) indicates the limit of the number of blood donation houses open. Equation (10) implies that only when the blood donation house $j$ is open can a donor group $i$ go for donation. Equations (11) and (12) suggest that if group $i$ does not donate blood to a blood donation house $j$, no blood donated by group $i$ will be sent from $j$ to blood centre $k$. Equation (13) shows that group $i$ can donate only after reaching the blood centre $k$. Equation (14) indicates that the blood will not be delivered from the blood centre without the assignment of the hospital. Equations (15) and (16) imply that group $i$ will choose an institution within its acceptable distance for donation. Equation (17) demonstrates the constraint of blood centre inventory status update. Equation (18) indicates that the inventory of blood centre $k$ will not exceed the maximum storage capacity of $k$. Equation (19) provides the time limit for the blood centre to deliver blood to the hospital. Equation (20) assumes that the hospital has no storage capacity. Equation (21) expresses that the amount of blood delivered by the blood centre to the hospital at time $t$ will not exceed its inventory.

The above model is dynamic stochastic programming and needs specific data to test its feasibility in the next section.

\section{Numerical Analysis}

This section selects the 8.0 surface-wave magnitude (Ms) earthquake of Wenchuan in China for data analysis based on the model in Section 3, which caused severe damage to Wenchuan and its surrounding environment. As a major city in the vicinity, Chengdu undertook most of the medical treatment for victims. Hence, this research is based on relevant data of prefectures in Chengdu.

4.1. Data. According to the 2019 Statistical Yearbook of Chengdu issued on the public information website of Chengdu Bureau of Statistics, there are 20 municipal districts and county-level cities in Chengdu, as shown in Figure 2. Through comparison, it is found that only minor differences lie in its 2008 and 2019 administrative division maps. In this paper, the latest version of the administrative division map was selected to perform relevant calculations.

4.1.1. Supply Data. Blood donors are divided into different groups based on Chengdu administrative divisions. As a result, 20 blood donation groups were identified, denoted as $i=1,2, \ldots$, and 20, separately. Additionally, the administrative centre of each division was selected as the starting point for a group to donate blood.

After an emergency, all blood donation groups become active. They altruistically donate blood and help the disasteraffected people. According to statistics, the number of blood donors after an emergency is three times greater than usual. This work's research period lasted for 15 days, and corresponding investigations were made on a time frame of 1 day. After processing, the daily average number of blood donors since an emergency was obtained, that is, the mean values $(60,73,80,129,80,72,44,81,48,95,125,63,54,28,33,66$, $85,69,70,158)$ of blood donors in respective groups $i$. As assumed, the daily blood supply from a single group obeys the Poisson distribution. Hence, the blood volume donated can be calculated accordingly.

4.1.2. Demand Data. During the 12 May Wenchuan Earthquake, many hospitals in diverse districts and counties of Chengdu actively received and rescued patients transferred from the affected area. Among them, nine major hospitals were selected for this research, including Sichuan Provincial People's Hospital. Apart from numbering these hospitals, their mean values of demand are listed in Table 4 $[41,42]$.

It is assumed that the daily demand, $D_{h t}$, of each hospital abides by the Poisson distribution that considers the above data as the mean values. Under the circumstance that random numbers from the Poisson distribution can be generated based on these mean values, specific demands of the hospitals were acquired.

4.1.3. Distance Data. The Chengdu Blood Centre, denoted by $k$, is situated at Wuhou District, Chengdu City. It has multiple blood donation houses. To simplify calculations, 10 


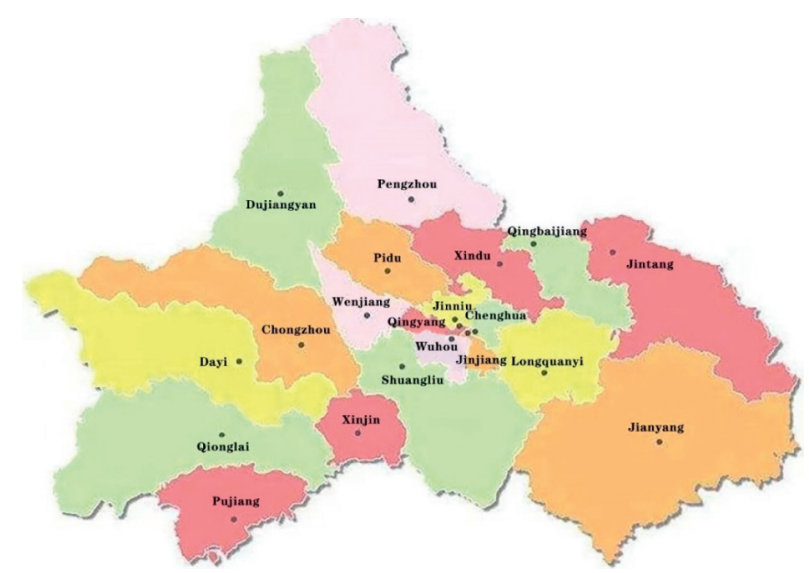

FIgURe 2: Administrative division map of Chengdu (the 2019 Statistical Yearbook of Chengdu).

TABLE 4: Mean values of blood demand (unit: U).

\begin{tabular}{lccccc}
\hline Serial numbers of hospitals & 1 & 2 & 3 & 4 & 5 \\
\hline Blood demand & 289 & 126 & 52 & 48 & 46 \\
Serial numbers of hospitals & 6 & 7 & 8 & 9 & \\
Blood demand & 28 & 25 & 21 & 20 & \\
\hline
\end{tabular}

blood donation houses were selected and numbered. Moreover, the distance from a blood donation house, $j$, to the Chengdu Blood Centre, $k$, was presented, where $j=1,2, \ldots$, and 10 , and $k=1$ (Table 5).

20 blood donation groups, $i$, donate RBCs at the blood donation house, $j$, or the blood centre, $k$, and the corresponding distance is shown in Tables 6 and 7.

The other parameters were set as follows. The research period $\mathrm{t}$ of the proposed model in this study is defined as 15 days. The initial inventory of Phase $1, I B_{k 1}$, is equal to $600 \mathrm{U}$ $(1 U=200 \mathrm{ml})$. The unit transportation cost of a vehicle is represented by $T J$, and it is set as $0.8 \mathrm{CNY} / 100$ units $/ \mathrm{km}$. When the blood volume is under 100 units $(1$ unit $=1 \mathrm{U})$, it is simply calculated as 100 units. WH is defined to be CNY 40/ $\mathrm{U} ; b$ is set as $600 \mathrm{U} ; c b$ means that storage capacity of the blood centre is $15,000 \mathrm{U}$; $q$, equal to 10 , refers to the total number of blood donation houses; $L T_{1}$ is set as $2.5 \mathrm{~h} ; A C$ signifies that the maximum acceptable distance from the centre to blood donation groups is $15 \mathrm{~km}$; and $V_{c}, 30 \mathrm{~km} / \mathrm{h}$, is the speed of a blood delivery vehicle.

\subsection{Numerical Results and Discussion}

4.2.1. Donation and Opening of Blood Donation Houses. Setting $\beta=0.1, w_{1}=0.8$, and $w_{2}=0.2$, the Poisson random number is generated according to the given average group supply and hospital demand to get the daily supply quantity of each group and the daily demand quantity of each hospital. In the following numerical analysis, ratio of apheresis donation $\beta$ is fluctuated and ranges from 0 to 1 . Weight coefficients $\left(w_{1} w_{2}\right)$ indicate the importance of the two objectives. Life safety is considered as the most
TABle 5: Distance $A_{j k}$ from the blood donation houses to the blood centres (unit: km).

\begin{tabular}{lccccc}
\hline $\begin{array}{l}\text { Serial numbers of blood donation } \\
\text { houses }\end{array}$ & 1 & 2 & 3 & 4 & 5 \\
\hline $\begin{array}{l}\text { Distance } \\
\begin{array}{l}\text { Serial numbers of blood donation } \\
\text { houses }\end{array}\end{array}$ & 65.5 & 63.8 & 63.4 & 36.2 & 26.5 \\
Distance & 24.2 & 44.3 & 82.5 & 45.3 & 59.9 \\
\hline
\end{tabular}

important task in this work. Hence, the weight coefficient of shortage penalty cost $\left(w_{1}\right)$ is given a much higher value, while the coefficient of the other cost is relatively low. Lingo is introduced to solve the model to obtain the number of open blood donation houses and blood collection in each period, as shown in Table 8 (the number outside the bracket is the serial number of the open blood donation house, and the serial number of numbers in the bracket corresponds to the amount of blood collected in the blood donation house).

When $t$ ranges from 1 to 14 , Group $i$ will choose blood centre $k$ or blood donation house $j$ for blood donation, and blood donation house $j$ will be open or closed. The blood collected at each stage will be added to the beginning inventory of the blood centre in the next stage.

As the results suggest, under the current data settings, not all the blood donation houses are required to be operational under the current data settings. If there is both a blood donation house and a blood centre within an acceptable distance, the blood centre will be preferred. Since the blood collected in the blood donation house has to be delivered to the blood centre, there are attendant transportation costs, in addition to the cost of opening the blood donation house.

4.2.2. Allocation Scheme. On account of the initial data settings in this section, the calculated allocation scheme is shown in Table 9.

Among them, the demands of 7 hospitals in period $t=1$ are all met, while the demands of red blood cells of hospitals 
TABle 6: Distance $A_{i j}$ from different groups to the blood donation house (unit: km).

\begin{tabular}{|c|c|c|c|c|c|c|c|c|c|c|}
\hline & 1 & 2 & 3 & 4 & 5 & 6 & 7 & 8 & 9 & 10 \\
\hline 1 & 23.7 & 71.7 & 71.3 & 39.8 & 34.5 & 24.5 & 55 & 93.5 & 47.3 & 59.1 \\
\hline 2 & 20.3 & 72.1 & 61.7 & 24.5 & 24.5 & 25.1 & 44.3 & 82.5 & 46.5 & 63.3 \\
\hline 3 & 25.5 & 69.6 & 60.2 & 22.6 & 23.3 & 21.6 & 44.3 & 83.5 & 48.5 & 65.5 \\
\hline 4 & 16 & 64.1 & 61.3 & 33.7 & 26.8 & 16.4 & 43.3 & 82 & 42.6 & 62.4 \\
\hline 5 & 29 & 65.1 & 64.7 & 28.6 & 27.8 & 26.6 & 51.5 & 86 & 51.7 & 60.4 \\
\hline 6 & 41.8 & 96.6 & 110 & 66.2 & 73 & 42 & 92.9 & 99.8 & 60.3 & 40.5 \\
\hline 7 & 67 & 78.3 & 101 & 56.5 & 54.7 & 67.1 & 84.6 & 122.6 & 90.5 & 72.3 \\
\hline 8 & 50 & 57.1 & 80.5 & 37 & 43.6 & 50 & 64.2 & 102.5 & 82.2 & 73.5 \\
\hline 9 & 16.7 & 49.3 & 43.9 & 17.7 & 2.9 & 16.9 & 25.5 & 63.7 & 35 & 81.3 \\
\hline 10 & 75.8 & 92.9 & 115.8 & 71 & 85 & 76 & 99 & 137.5 & 93.9 & 63 \\
\hline 11 & 16 & 63.1 & 47 & 33.3 & 19.6 & 1 & 30.5 & 58.7 & 23.6 & 74.3 \\
\hline 12 & 30 & 37.8 & 61 & 2.6 & 15.8 & 30 & 42.6 & 80.8 & 47.7 & 84.1 \\
\hline 13 & 46.6 & 76.8 & 1 & 59.6 & 45.3 & 46.6 & 22.2 & 22 & 38 & 119.3 \\
\hline 14 & 74.5 & 134.4 & 53.8 & 103.7 & 103 & 74.5 & 88 & 31.5 & 41.3 & 123.8 \\
\hline 15 & 23.3 & 82.7 & 38.7 & 65.5 & 38.2 & 23.3 & 33.2 & 37.3 & 0.5 & 85.4 \\
\hline 16 & 63.2 & 2.3 & 73.7 & 34.6 & 44.5 & 63.4 & 57 & 95 & 83 & 117.5 \\
\hline 17 & 60.1 & 34.3 & 76.6 & 22.7 & 37.3 & 60.3 & 60 & 98.2 & 86 & 102 \\
\hline 18 & 58 & 95 & 22.3 & 77.9 & 63.6 & 58.3 & 40.3 & 1 & 39.3 & 122.2 \\
\hline 19 & 28.4 & 56.8 & 22.6 & 39.6 & 25.4 & 30 & 1 & 39.6 & 32.9 & 100.9 \\
\hline 20 & 73.9 & 116.8 & 117.8 & 86.2 & 83.7 & 73.8 & 100.5 & 122.1 & 86.2 & 2.9 \\
\hline
\end{tabular}

TABle 7: Distance $A_{i k}$ from different donation groups to the blood centre (unit: $\mathrm{km}$ ).

\begin{tabular}{ccccccccccc}
\hline & 1 & 2 & 3 & 4 & 5 & 6 & 7 & 8 & 9 & 10 \\
\hline $\mathrm{K}$ & 12.7 & 3.1 & 7.2 & 4.4 & 4.4 & 24.5 & 34.4 & 24 & 24.4 & 49.5 \\
$\mathrm{~K}$ & 11 & 12 & 13 & 14 & 15 & 16 & 17 & 18 & 19 & 20 \\
$\mathrm{~K}$ & 25.8 & 25.6 & 64.5 & 83.3 & 45.3 & 62.6 & 43 & 82.8 & 44.5 & 59.1 \\
\hline
\end{tabular}

TABle 8: Number of blood donation houses open and blood collected in each period.

\begin{tabular}{|c|c|c|c|}
\hline \multirow{2}{*}{$\begin{array}{l}\text { Period } t \\
1\end{array}$} & \multicolumn{2}{|c|}{$\begin{array}{c}\text { Opening status of } \\
\text { blood donation } \\
\text { houses }\end{array}$} & \multirow{2}{*}{$\frac{\text { Number of blood donation houses }}{2}$} \\
\hline & $5(56)$ & $10(153)$ & \\
\hline 2 & $10(180)$ & - & 1 \\
\hline 3 & $1(115)$ & $10(103)$ & 2 \\
\hline 4 & $10(170)$ & - & 1 \\
\hline 5 & 10 (147) & - & 1 \\
\hline 6 & $10(177)$ & - & 1 \\
\hline 7 & $10(163)$ & - & 1 \\
\hline 8 & $6(127)$ & $7(71)$ & 2 \\
\hline 9 & $4(88)$ & 10 (158) & 2 \\
\hline 10 & $10(173)$ & - & 1 \\
\hline 11 & $10(163)$ & - & 1 \\
\hline 12 & $8(70)$ & 10 (177) & 2 \\
\hline 13 & 10 (185) & - & 1 \\
\hline 14 & $1(143)$ & $8(72)$ & 2 \\
\hline
\end{tabular}

numbered 5 and 7 fail to be fully responded. In the period $t=1$, the shortage of red blood cells is $27 \mathrm{U}$.

4.2.3. Optimal Distribution of Costs. Table 10 shows details of the optimal distribution of each cost in the blood supply chain.
TABle 9: Hospital demand and allocation of red blood cells in period $t=1$ (unit: $\mathrm{U}$ ).

\begin{tabular}{lccccc}
\hline Serial number & 1 & 2 & 3 & 4 & 5 \\
\hline Quantity demanded & 269 & 137 & 46 & 42 & 46 \\
Quantity allocated & 269 & 137 & 46 & 42 & 42 \\
Serial number & 6 & 7 & 8 & 9 & \\
Quantity demanded & 26 & 23 & 21 & 17 & \\
Quantity allocated & 26 & 0 & 21 & 17 & \\
\hline
\end{tabular}

TABLE 10: Details of the comprehensive costs of the blood supply chain (unit: CNY).

\begin{tabular}{lcc}
\hline Shortage cost & $\begin{array}{c}\text { Opening cost of blood } \\
\text { donation houses }\end{array}$ & Operating cost \\
\hline 81,000 & 60,000 & $2,185,282$ \\
Transportation & Inventory cost & $\begin{array}{c}\text { Optimal } \\
\text { objective }\end{array}$ \\
cost & 5,160 & 515,707 \\
\hline
\end{tabular}

4.3. Sensitivity Analysis. The following is an exploration of the sensitivity of the parameters on the optimal objective and optimal decisions.

4.3.1. Different Supply States. The mean of supply in the example is based on the expectation of a higher level of enthusiasm for donation in an emergency, whereas the reality might be more positive or negative than expected. The product of the mean of supply and the multiple can test the impacts of supply states on the scheme, where the multiple ranges from 0.1 to 1.5 .

As shown in Figure 3, the shortage of red blood cells is negatively correlated with the average supply, while the opening cost of blood donation houses is also negatively correlated with the supply quantity, with a decline in the 


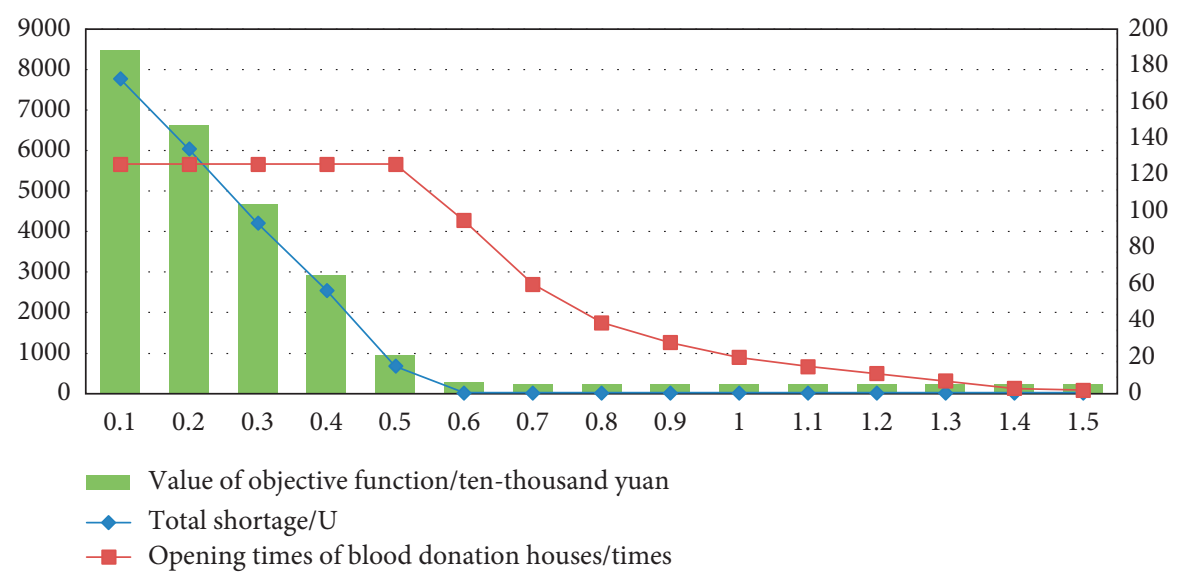

FIgURE 3: Shortage and opening status of blood donation houses.

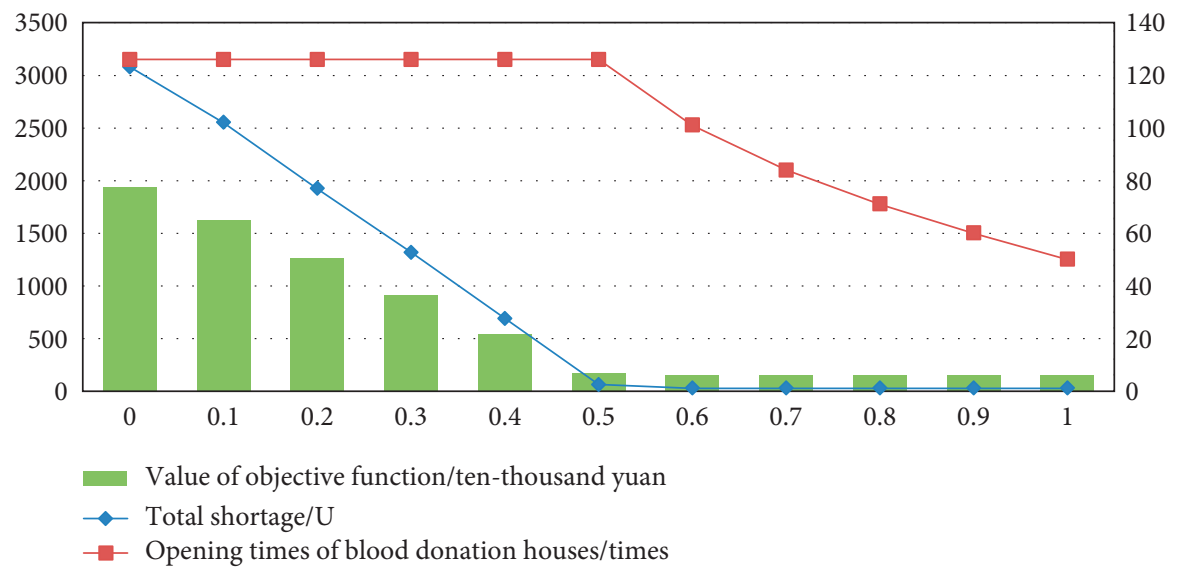

FIgURE 4: Impact of fluctuations in proportion of apheresis donation.

objective function value. This is because when the average supply is low, there is less supply and more shortage. As the mean value increases, the shortage of red blood cells decreases. Where the supply fails to meet the demand, a larger number of blood donation houses will be open for blood collection. As the supply increases, the need for blood donation houses reduces. Therefore, the opening times and costs of blood donation houses decrease with the increase of supply.

\subsubsection{Fluctuations in Proportion of Apheresis Donation.} The ratio of apheresis donation $\beta$ is fluctuated, and the value of $\beta$ ranges from 0 to 1 . Changes in $\beta$ demonstrate a slight effect on the scheme and the quantity in short, as calculated by the original supply and demand quantity. In this section, 0.4 times of the initial supply mean is taken as the new supply mean before generating the supply data through Poisson distribution. The demand data remain unchanged. The influence of changes in the value of $\beta$ on the shortage quantity is as shown in Figure 4.

With the increase of $\beta$, there is a dramatic decrease in the number of red blood cell shortage. The reason is that the number of red blood cells donated by single donors in the form of apheresis is twice the number of red blood cells donated by whole blood. When $\beta$ ranges from 0.6 to 1 , the total amount of shortage remains $27 \mathrm{U}$. This is because there will be no shortage since the second period, and the red blood cell shortage is related to the initial inventory setting of the first period when the supply quantity meets the demand. The opening times of blood donation houses remain unchanged when the value of $\beta$ ranges from 0 to 0.5 and then decrease with the increase of the value of $\beta$. The corresponding reason is that the supply quantity meets the demand from the second period when the value of $\beta$ ranges from 0.6 to 1 . Since it is no longer necessary to open all blood donation houses to respond to blood collection demands, the total number of opening times of blood donation houses is reduced. The value of objective function decreases with the increase of the value of $\beta$.

Therefore, increasing the ratio of apheresis donation will in a way reduce shortage and the value of objective function.

4.3.3. Limit of Donation times. Based on the initial data settings, by adjusting the limit of donation times, the influence of changes in donation times on the scheme is 


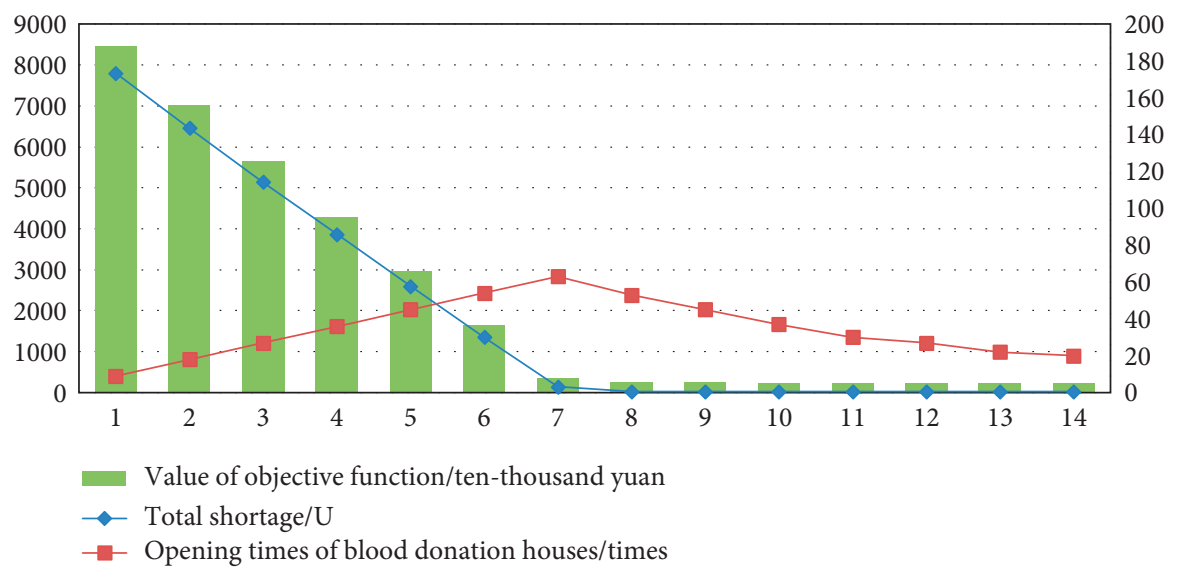

FIGURE 5: Results of different donation time limits.

TABLE 11: Impact of AC distance change on red blood cell shortage.

\begin{tabular}{lcccc}
\hline $\begin{array}{l}\text { AC distance limits } \\
(\mathrm{km})\end{array}$ & $\begin{array}{c}\text { Total red blood cell } \\
\text { shortage }(\mathrm{U})\end{array}$ & $\begin{array}{c}\text { Opening times of blood donation } \\
\text { houses (times) }\end{array}$ & $\begin{array}{c}\text { Serial number of newly } \\
\text { included group } i\end{array}$ & $\begin{array}{c}\text { Value of objective } \\
\text { function }(\mathrm{CNY})\end{array}$ \\
\hline 15 & 2551 & 126 & - & 6517748 \\
20 & 2551 & 98 & $6,8 、 17$ & 6500434 \\
25 & 1100 & 70 & - & 3069429 \\
30 & 1100 & 70 & $7 、 14$ & 3069483 \\
35 & 672 & 56 & - & 2054066 \\
40 & 672 & 42 & - & 2045611 \\
45 & 672 & 42 & 10 & 2045400 \\
50 & 104 & 42 & & 711645 \\
\hline
\end{tabular}

discussed in this section. Figure 5 shows shortage of red blood cells and changes in the number of blood donation houses with different donation time limits.

As shown in Figure 5, there is a notable decrease in the shortage of red blood cells and the value of objective function with the relaxation of donation time limits for blood donors during the research period. The number of opening blood donation houses first increases and then decreases. The reason is that some people, at a great donation time limit, are not allowed to donate blood more than once in the research period, which leads to the unnecessary opening of blood donation houses and the decrease of opening times. However, with the relaxation of donation time limits, larger groups of people will have access to blood donation, resulting in an increase in the number of donations available and the opening times of blood donation houses. When the donation time limit is relaxed to such a level (for instance, seven times or more) that the blood supply meets demands, the number of blood donation houses will be reduced.

4.3.4. Changes in AC Distance. In this section, 0.4 times of the initial supply mean is taken as the new supply mean before generating the supply data through Poisson distribution. The demand data, however, remained unchanged.

Table 11 shows the number of red blood cell shortage in blood donation distance of $15-50 \mathrm{~km}$, the maximum number of opening blood donation houses in each period, and the newly included blood donor groups after distance expansion.

As implied in Table 11, the shortage of red blood cells falls with the rise of people's acceptable distance after distance expansion. Some new blood donors are enrolled, and the number of blood donation houses required is decreasing. The cost of opening blood donation houses is reduced because some groups go to the same donation houses or to a blood centre within an acceptable distance after distance expansion.

\section{Conclusions and Future Research}

5.1. Conclusions. This study focuses on blood supply chain operation-related problems in emergencies. It proposed a four-echelon blood supply chain model consisting of blood donors, blood donation houses, blood centres, and hospitals. By virtue of the proposed model, this study attempts to minimise the composite costs including the shortage cost incurred in blood centres, the cost of opening a blood donation house, and the corresponding operating cost. Through numerical analysis, the feasibility of the model is verified and the following conclusions are drawn.

(1) From the holistic perspective of supply chain, a stochastic optimization-based blood supply chain model is constructed. Simultaneously, it takes blood 
collection and supply problems in a blood supply chain into account. Lingo is introduced to solve the model to find the optimal corresponding operation scheme of the blood supply chain, such as the decisions on when and where blood donation houses should be open, how many blood donation houses should be open at a time, how much blood should be collected, how blood centres should distribute blood to hospitals in disaster areas, and so on. Furthermore, the sensitivity of the parameters of the blood supply chain (e.g., proportion of RBC apheresis donation, average of blood supply, times of blood donation, and distance between blood donors and blood facilities) is analysed to test the impacts of various parameter settings on the scheme. Thus, it is suggested that related decision makers need to get a feasible operational scheme by setting relevant parameters according to the actual situation.

(2) By changing the average blood supply, the blood shortage situation is alleviated due to an increase in the average supply. The opening times of blood donation houses and comprehensive costs are also negatively correlated with the supply quantity. Hence, the corresponding demands can be satisfied without opening more blood donation houses.

Therefore, blood donation should be encouraged through effective publicity and transparency in information sharing. In this regard, it is suggested that relevant decision makers should formulate contingency plans and publicity mechanisms for high-risk emergencies in the region prior to emergencies, take timely measures to enhance residents' willingness for blood donation in case of insufficient supply quantity, in order to reduce the threat of quantity shortage, and adjust the opening of blood donation houses and blood collection methods to reduce the overall cost.

(3) Based on fluctuations in the proportion of blood apheresis collection, it is assumed that an increase in this proportion results in a significant decrease of the total RBC shortage and the RBC shortage cost. If this proportion rises, the number of blood donation houses in service can be lowered somewhat. In other words, the blood demand can be satisfied without opening all blood donation houses. Additionally, blood supply shortages caused by overuses in the early phases of an emergency are also eased (donors who have donated blood are forbidden from donating again within a short time).

Considering this, there is a need to encourage the apheresis collection of RBCs. It may be important to continuously innovate the technology of red blood cell apheresis collection, appropriately raise the proportion of apheresis blood donation, in order to increase the number of red blood cells donated by unit donors and relieve blood shortage out of the requirement for donation interval, and increase blood collection in the short term to reduce the opening cost of blood donation houses.

(4) As shown by modifying the distance acceptable to donors, it is found that some new blood donors are enrolled to donate as the distance increases. If other parameters remain unchanged, an increase in this distance leads to a rise in the blood volume available. When the average blood supply is not high, encouraging blood donors (particularly loyal blood donation populations) to increase their acceptable distance to blood facilities may enable the blood supply to go up, thus easing the blood shortage situation. Considering that different blood donation populations may select the same blood donation house, the total number of opening times of blood donation houses is lowered as well. Alternatively, donors may donate blood in a blood centre within their acceptable distance rather than in a blood donation house. As a result, the cost incurred by opening a great number of blood donation houses is reduced.

In this case, emergency management and blood departments are expected to provide convenience for blood donors to expand their donation scope through special transfers or rewards, thereby promoting them to expand their donation distance, increasing blood supply within the jurisdiction to relieve the supply pressure, and reducing the opening times and overall costs of blood donation houses.

(5) Proper adjustment of the donation frequency of donor groups is necessary. Given the time interval requirement for blood donations, a group that made multiple blood donations over the research period implies a significant reduction in the number of subsequent donations. Nevertheless, secondary disasters associated with emergencies frequently bring about casualties, triggering a renewed rise in blood demand. A substantial decline in the number of donations within the early acceptable distances may result in a supply shortfall.

To increase the total amount of available blood and eliminate the risk of a blood shortage, it is recommended to encourage a greater number of donors to be involved in blood collection, conduct red blood cell collection in the form of apheresis processes, increase the ratio of apheresis collection through technical research and relevant investment and training, and promote donors to extend their donation distance to increase the number of available donor groups, thereby enhancing blood quantity and reducing the risk of a blood shortage. Moreover, it is suggested to properly relax the donation time limits for blood donors to alleviate a blood shortage due to excessive short-term donations. These four measures are expected to work best through a combination of them.

5.2. Future Research. There also exist some potential issues for the future study. First, mobile blood donation sites are 
also important channels for blood collection and recommended to be incorporated in the future blood supply chain. Second, blood types and other indexes are suggested to be incorporated into subsequent investigations to explore blood supply chain operations further.

\section{Data Availability}

The data used to support the findings of this study are included within the article.

\section{Conflicts of Interest}

The authors declare that there are no conflicts of interest regarding the publication of this paper.

\section{Acknowledgments}

This study was supported by the "Three Levels" Talent Construction Project of Zhuhai College of Science and Technology, the 2018 Discipline Co-Construction Project of the 13th Five-Year Plan of Philosophy and Social Sciences of Guangdong Province (grant no. GD18XTS06), the $13^{\text {th }}$ FiveYear Plan of Philosophy and Social Sciences of Guangdong Province (grant no. GD18XGL24), and the Department of Science and Technology of Guangdong Province (grant no. 2020A0505090004).

\section{References}

[1] M. Dillon, F. Oliveira, and B. Abbasi, "A two-stage stochastic programming model for inventory management in the blood supply chain," International Journal of Production Economics, vol. 187, pp. 27-41, 2017.

[2] A. Pirabán, W. J. Guerrero, and N. Labadie, "Survey on blood supply chain management: models and methods," Computers \& Operations Research, vol. 112, p. 104756, 2019.

[3] H. Tang, A. Elalouf, E. Levner, and T. C. E. Cheng, "Efficient computation of evacuation routes on a three-dimensional geometric network," Computers \& Industrial Engineering, vol. 76, pp. 231-242, 2014.

[4] J. H. Zhang, H. Y. Liu, R. Zhu, and L. Yang, "Emergency evacuation of hazardous chemical accidents based on diffusion simulation," Complexity, vol. 2017, Article ID 4927649, 16 pages, 2017.

[5] Z. Liu, X. Li, and X. Chen, "Evacuation traffic management under diffusion of toxic gas based on an improved road risk level assessment method," Complexity, vol. 2019, Article ID 6768526, 11 pages, 2019.

[6] J. Ray, A Multi-Period Linear Programming Modal for Optimally Scheduling the Distribution of Food-Aid in West Africa, University of Tennessee, Knoxville, TN, USA, 1987.

[7] A. Rathi, R. Church, and R. Solanki, "Allocating resources to support a multicommodity flow with time windows," Logistics \& Transportation Review, vol. 28, no. 2, pp. 167-188, 1992.

[8] A. Haghani and S.-C. Oh, "Formulation and solution of a multi-commodity, multi-modal network flow model for disaster relief operations," Transportation Research Part A: Policy and Practice, vol. 30, no. 3, pp. 231-250, 1996.

[9] L. Özdamar, E. Ekinci, and B. Küçükyazici, "Emergency logistics planning in natural disasters," Annals of Operations Research, vol. 129, no. 1-4, pp. 217-245, 2004.
[10] W. Yi and L. Özdamar, "A dynamic logistics coordination model for evacuation and support in disaster response activities," European Journal of Operational Research, vol. 179, no. 3, pp. 1177-1193, 2007.

[11] W. Yi and A. Kumar, "Ant colony optimization for disaster relief operations," Transportation Research Part E: Logistics and Transportation Review, vol. 43, no. 6, pp. 660-672, 2007.

[12] J.-B. Sheu, "Dynamic relief-demand management for emergency logistics operations under large-scale disasters," Transportation Research Part E: Logistics and Transportation Review, vol. 46, no. 1, pp. 1-17, 2010.

[13] S. Yan, C.-K. Lin, and S.-Y. Chen, "Logistical support scheduling under stochastic travel times given an emergency repair work schedule," Computers \& Industrial Engineering, vol. 67 , no. 1 , pp. 20-35, 2014.

[14] V. Bosnes, M. Aldrin, and H. E. Heier, "Predicting blood donor arrival," Transfusion, vol. 45, no. 2, pp. 162-170, 2005.

[15] A. F. Osorio, S. C. Brailsford, and H. K. Smith, "Whole blood or apheresis donations? A multi-objective stochastic optimization approach," European Journal of Operational Research, vol. 266, no. 1, pp. 193-204, 2018.

[16] B. Seda, C. Giuliana, L. Ettore, and Y. Semih, "An appointment scheduling framework to balance the production of blood units from donation," European Journal of Operational Research, vol. 265, no. 3, pp. 1124-1143, 2018.

[17] L. A. Williams, J. Sun, and B. Masser, "Integrating self-determination theory and the theory of planned behaviour to predict intention to donate blood," Transfusion Medicine, vol. 29, no. S1, pp. 59-64, 2019.

[18] L. Goette and A. Stutzer, "Blood donations and incentives: evidence from a field experiment," Journal of Economic Behavior \& Organization, vol. 170, pp. 52-74, 2020.

[19] N. M. Heddle, Y. Liu, R. Barty et al., "Factors affecting the frequency of red blood cell outdates: an approach to establish benchmarking targets," Transfusion, vol. 49, no. 2, pp. 219-226, 2009.

[20] L. Zheng and C. Xu, "Blood order and collection problems with two demand classes and emergency replenishment," Journal of the Operational Research Society, vol. 72, no. 3, pp. 501-518, 2021.

[21] G. P. Prastacos, "Optimal myopic allocation of a product with fixed lifetime," Journal of the Operational Research Society, vol. 29, no. 9, pp. 905-913, 1978.

[22] G. Sahin, S. Süral, and S. Meral, "Locational analysis for regionalization of Turkish red crescent blood services," Computers \& Operations Research, vol. 34, no. 3, pp. 692-704, 2007.

[23] P. Sivakumar, K. Ganesh, and P. Parthiban, "Multi-phase composite analytical model for integrated allocation-routing problem - application of blood bank logistics," International Journal of Logistics Economics and Globalisation, vol. 1, no. 3/ 4, pp. 251-281, 2008.

[24] S. Gunpinar and G. Centeno, "An integer programming approach to the bloodmobile routing problem," Transportation Research Part E: Logistics and Transportation Review, vol. 86, pp. 94-115, 2016.

[25] K.-M. Wang and Z.-J. Ma, "Age-based policy for blood transshipment during blood shortage," Transportation Research Part E: Logistics and Transportation Review, vol. 80, pp. 166-183, 2015.

[26] M. Dehghani and B. Abbasi, "An age-based lateral-transshipment policy for perishable items," International Journal of Production Economics, vol. 198, pp. 93-103, 2018. 
[27] Y. Sha and J. Huang, "The multi-period location-allocation problem of engineering emergency blood supply systems," Systems Engineering Procedia, vol. 5, no. 1, pp. 21-28, 2012.

[28] B. Fahimnia, A. Jabbarzadeh, A. Ghavamifar, and M. Bell, "Supply chain design for efficient and effective blood supply in disasters," International Journal of Production Economics, vol. 183, pp. 700-709, 2017.

[29] R. Ramezanian and Z. Behboodi, "Blood supply chain network design under uncertainties in supply and demand considering social aspects," Transportation Research Part E: Logistics and Transportation Review, vol. 104, pp. 69-82, 2017.

[30] O. Ö. Özener, A. Ekici, and E. Çoban, "Improving blood products supply through donation tailoring," Computers \& Operations Research, vol. 102, pp. 10-21, 2019.

[31] B. Hamdan and A. Diabat, "A two-stage multi-echelon stochastic blood supply chain problem," Computers \& Operations Research, vol. 101, pp. 130-143, 2019.

[32] M. R. G. Samani, S. M. Hosseini-Motlagh, and S. Homaei, "A reactive phase against disruptions for designing a proactive platelet supply network," Transportation Research Part E: Logistics and Transportation Review, vol. 140, Article ID 102008, 2020

[33] A. Jabbarzadeh, B. Fahimnia, and S. Seuring, "Dynamic supply chain network design for the supply of blood in disasters: a robust model with real world application," Transportation Research Part E: Logistics and Transportation Review, vol. 70, pp. 225-244, 2014.

[34] M. Arvan, R. Tavakoli-Moghadam, and M. Abdollahi, "Designing a bi-objective and multi-product supply chain network for the supply of blood," Uncertain Supply Chain Management, vol. 3, no. 1, pp. 57-68, 2015.

[35] A. Haeri, S. M. Hosseini-Motlagh, M. R. Ghatreh Samani, and M. Rezaei, "A mixed resilient-efficient approach toward blood supply chain network design," International Transactions in Operational Research, vol. 27, no. 4, pp. 1962-2001, 2020.

[36] W. Q. Liu, G. Y. Ke, J. Chen, and L. M. Zhang, "Scheduling the distribution of blood products: a vendor-managed inventory routing approach," Transportation Research Part E: Logistics and Transportation Review, vol. 140, Article ID 101964, 2020.

[37] C. Wang and S. Chen, "A distributionally robust optimization for blood supply network considering disasters," Transportation Research Part E: Logistics and Transportation Review, vol. 134, Article ID 101840, 2020.

[38] B. Zahiri and M. S. Pishvaee, "Blood supply chain network design considering blood group compatibility under uncertainty," International Journal of Production Research, vol. 55, no. 7, pp. 2013-2033, 2017.

[39] M. Y. N. Attari and E. N. Jami, "Robust stochastic multichoice goal programming for blood collection and distribution problem with real application," Journal of Intelligent \& Fuzzy Systems, vol. 35, no. 2, pp. 2015-2033, 2018.

[40] M. R. G. Samani and S.-M. Hosseini-Motlagh, "An enhanced procedure for managing blood supply chain under disruptions and uncertainties," Annals of Operations Research, vol. 283, no. 1-2, pp. 1413-1462, 2019.

[41] Z. J. Ma, Theory and Method of Blood Support for Unconventional Emergencies, Science Press, Beijing, China, 2015.

[42] Z. J. Ma, Investigation Of Blood Relief In "5.12" Wenchuan Earthquake, Southwest Jiaotong University, Chengdu, China, 2010. 